\title{
DETERMINATION OF SURFACES IN THREE-DIMENSIONAL MINKOWSKI AND EUCLIDEAN SPACES BASED ON SOLUTIONS OF THE SINH-LAPLACE EQUATION
}

\author{
PAUL BRACKEN \\ Received 23 January 2005
}

The relationship between solutions of the sinh-Laplace equation and the determination of various kinds of surfaces of constant Gaussian curvature, both positive and negative, will be investigated here. It is shown that when the metric is given in a particular set of coordinates, the Gaussian curvature is related to the sinh-Laplace equation in a direct way. The fundamental equations of surface theory are found to yield a type of geometrically based Lax pair for the system. Given a particular solution of the sinh-Laplace equation, this Lax can be integrated to determine the three fundamental vectors related to the surface. These are also used to determine the coordinate vector of the surface. Some specific examples of this procedure will be given.

The relationship between the solutions of certain types of partial differential equations and the determination of various kinds of surfaces of constant curvature has generated many results which have applications to the areas of both pure and applied mathematics. This includes the determination of surfaces of either constant mean curvature or Gaussian curvature. It has long been known that there is a connection between surfaces of negative constant Gaussian curvature in Euclidean $\mathbb{R}^{3}$ and the sine-Gordon equation. Moreover, a great deal of work has been done recently $[1,3]$ with regard to the generalized Weierstrass representation which permits inducing surfaces of constant mean curvature in $\mathbb{R}^{3}$ as well as in higher-dimensional Euclidean and non-Euclidean spaces. The partial differential equations whose solutions are used to generate surfaces in these types of spaces are known to have soliton solutions and many examples of these have been given [2]. From these types of solutions, it is usually possible to construct multisoliton solutions for the equation. This can often be accomplished in more than one way. For example, an auto-Bäcklund transformation can be determined for the differential equation, or a system of differential equations, by making use of the transformation itself, or by making direct use of the theorem of permutability [4].

We introduce here some fundamental concepts and equations pertaining to the theory of surfaces in three-space, and, in particular, we study a class of sinh-Laplace equation 
which has the form

$$
\frac{\partial^{2} u}{\partial x^{2}}+\frac{\partial^{2} u}{\partial t^{2}}= \pm \sinh u
$$

Some further results are obtained based on the fundamental equations of surface theory, and it is shown how specific solutions of the sinh-Laplace equation (1) can be used in integrating these results to obtain the coordinates of a surface in either Minkowski $\mathbb{R}^{2,1}$ or Euclidean $\mathbb{R}^{3}$ space $[5,6]$. For future reference, $S^{2}$ will be the unit sphere in Euclidean space $\mathbb{R}^{3}, H^{2}$ and $S^{1,1}$ are the unit "spheres" in the Minkowski space $R^{2,1}$ with curvature -1 and +1 , respectively. It will be noted that there is a close relationship between (1) and the harmonic maps from $\mathbb{R}^{2}$ to $S^{2}$. From the harmonic maps, the Lax pair with this integrability condition can be obtained, and conversely, from each solution of (1), the corresponding harmonic map can be constructed. In fact, harmonic maps have many applications. The study of Yang-Mills theories has been motivated in part by analogies with general relativity. Harmonic maps model, in a simplified form, a type of nonlinearity that occurs in the Einstein equations, but different from that modeled by Yang-Mills theories. Gauge vector fields, or connections in bundles, can be defined by using solutions of the harmonic mapping equations, instead of the more familiar, inequivalent, Yang-Mills equations. In any case, producing connections in bundles has applications to both pure and applied areas of mathematics.

We now begin by introducing some basic ideas from differential geometry of surfaces. Let $\mathbf{r}$ be the position vector of point $P$ in three-space such that $\mathbf{r}=\mathbf{r}\left(u^{1}, u^{2}\right)$ is the parametrization of a surface, and $\left\{P, \mathbf{e}_{1}, \mathbf{e}_{2}, \mathbf{n}\right\}$ a frame with $P$ as its origin, $\mathbf{e}_{a}=\partial \mathbf{r} / \partial u^{a}$ and $\mathbf{n}$ the normal vector. It is possible to take linear combinations of the vectors $\mathbf{e}_{1}, \mathbf{e}_{2}$ to produce an orthonormal set $\mathbf{f}_{1}, \mathbf{f}_{2}$. However, the notation will not be altered and the basis $\left\{\mathbf{e}_{1}, \mathbf{e}_{2}, \mathbf{n}\right\}$ is simply referred to as an orthonormal frame for the surface. Writing the differentials of $\mathbf{r}, \mathbf{e}_{a}$, and $\mathbf{n}=\mathbf{e}_{3}$ as linear combinations of the vectors in this set, the fundamental equations of the theory of surfaces are written as

$$
\begin{gathered}
d \mathbf{r}=\omega^{a} \mathbf{e}_{a}, \\
d \mathbf{e}_{a}=\omega_{a}^{b} \mathbf{e}_{b}+\omega_{a}^{3} \mathbf{n}, \quad a, b=1,2, \\
d \mathbf{n}=\omega_{3}^{b} \mathbf{e}_{b} .
\end{gathered}
$$

In (2), $\omega_{b}^{a}, \omega_{a}^{3}$ are differential one-forms which depend on $u^{1}, u^{2}$ and satisfy

$$
\omega_{b}^{a}=-\omega_{a}^{b}, \quad \omega_{3}^{a}=\omega_{a}^{3} .
$$

The first fundamental form of the surface is

$$
I=d \mathbf{r} \cdot d \mathbf{r}=g_{a b} \omega^{a} \omega^{b}, \quad g_{a b}=\mathbf{e}_{a} \cdot \mathbf{e}_{b}=g_{b a} .
$$

Differentiating the orthonormality condition $\mathbf{n} \cdot \mathbf{e}_{a}=0$, we obtain $\mathbf{n} \cdot d \mathbf{e}_{a}+\mathbf{e}_{a} \cdot d \mathbf{n}=0$. Upon substituting differentials from (2), this implies that

$$
\omega_{a}^{3}=-g_{a b} \omega_{3}^{b} .
$$


Writing $\omega_{a}^{3}=h_{a b} \omega^{b}$, then from $d^{2} \mathbf{r}=0$, it follows that $h_{a b}=h_{b a}$. The related form

$$
I I=-d \mathbf{r} \cdot d \mathbf{n}=-g_{a b} \omega_{3}^{b} \omega^{a}=h_{a b} \omega^{a} \omega^{b}
$$

is called the second fundamental form of the surface. Now $\omega_{a}^{b}$ can be written in the form

$$
\omega_{a}^{b}=\Gamma_{a c}^{b} d u^{c}
$$

where $\omega^{c}=d u^{c}$ and $\Gamma_{a c}^{b}$ are the Christoffel symbols. Differentiating $d \mathbf{r}$, we obtain

$$
d^{2} \mathbf{r}=\omega_{a}^{b} \wedge \omega^{a} \mathbf{e}_{b}+\omega_{a}^{3} \wedge \omega^{a} \mathbf{n}
$$

Since $\mathbf{e}_{a}$ and $\mathbf{n}$ are independent, this will vanish provided that

$$
\omega_{a}^{b} \wedge \omega^{a}=0, \quad \omega_{a}^{3} \wedge \omega^{a}=0 .
$$

Of course, we can take $\omega^{a}=d u^{a}$ and replace this in these equations. Upon differentiating $d \mathbf{e}_{a}$, we obtain

$$
d^{2} \mathbf{e}_{a}=\left(\omega_{b}^{c} \wedge \omega_{a}^{b}+d \omega_{a}^{c}+\omega_{3}^{c} \wedge \omega_{a}^{3}\right) \mathbf{e}_{c}+\left(\omega_{b}^{3} \wedge \omega_{a}^{b}+d \omega_{a}^{3}\right) \mathbf{n}=0 .
$$

The vanishing of this expansion implies that the following equations hold:

$$
\begin{gathered}
d \omega_{a}^{c}+\omega_{b}^{c} \wedge \omega_{a}^{b}+\omega_{3}^{c} \wedge \omega_{a}^{3}=0, \\
d \omega_{a}^{3}+\omega_{b}^{3} \wedge \omega_{a}^{b}=0 .
\end{gathered}
$$

Equations (11) and (12) are called the Gauss equation and the Codazzi equation, respectively. Finally, evaluating $d^{2} \mathbf{n}$ simply reproduces (12) and (9) once again. Writing (11) in the form $d \omega_{a}^{c}+\omega_{b}^{c} \wedge \omega_{a}^{b}=\omega_{a}^{3} \wedge \omega_{3}^{c}$, the left-hand side is denoted by

$$
d \omega_{a}^{b}+\omega_{c}^{b} \wedge \omega_{a}^{c}=\frac{1}{2} R_{a c d}^{b} \omega^{c} \wedge \omega^{d} .
$$

This serves to introduce the Riemann curvature tensor. Using (5), it will be seen that it can be related to the components $h_{a b}$ through the relation

$$
R_{a b c d}=h_{a c} h_{b d}-h_{b c} h_{a d}
$$

where $a, b, c, d$ take the values 1,2 . One of the reasons that it is possible to produce connections between surfaces and integrable equations, such as the sine-Gordon equation, or system (1), is that there is a very straightforward connection between the Riemann tensor and the Gaussian curvature $K$ of the surface,

$$
\frac{R_{1212}}{g_{11} g_{22}-g_{12}^{2}}=\frac{h_{11} h_{22}-h_{12}^{2}}{g_{11} g_{22}-g_{12}^{2}}=K .
$$

A second reason is that a parametrization of the surface can be written down such that the first fundamental form assumes a particular form in terms of a function which satisfies the sinh-Laplace equation in these specific coordinates. This leads to the following definition. 
Definition 1. A coordinate system $(t, x)$ of the space-like surface $S$ is called Tschebyscheff if the metric of $S$ takes the form

$$
I=\cosh ^{2} \frac{\alpha}{2} d t^{2}+\sinh ^{2} \frac{\alpha}{2} d x^{2},
$$

and the second fundamental form is given by

$$
I I=\cosh \frac{\alpha}{2} \sinh \frac{\alpha}{2}\left(d t^{2}+d x^{2}\right) .
$$

THeOREm 2. If a space-like surface $S$ is of constant curvature $K= \pm 1$ and free of umbilics, then (i) $S$ can be covered by charts with Tschebyscheff coordinates, (ii) the function $\alpha(t, x)$ in terms of Tschebyscheff coordinates satisfies the sinh-Laplace equation.

Proof. Let $\left\{P, \mathbf{e}_{i}\right\}$ with $i=1,2,3$ be a field of orthogonal frames of $S$ such that $\mathbf{e}_{1}, \mathbf{e}_{2}$ are unit tangent vectors to the lines of curvature and $\mathbf{e}_{3}$ is the unit normal vector to the surface. The fundamental equations of the surface are given by (2). Since $\mathbf{e}_{1}, \mathbf{e}_{2}$ are principal tangent vectors, we can write

$$
\omega_{1}^{3}=A \omega^{1}, \quad \omega_{2}^{3}=C \omega^{2} .
$$

Using the property that, in this case, the curvature of $S$ is $K=-1$, it must be that $A C=1$. Choose coordinates such that

$$
\omega^{1}=\chi d t, \quad \omega^{2}=\psi d x
$$

and put

$$
\omega_{2}^{1}=-\omega_{1}^{2}=\xi d t+\eta d x
$$

where $\xi$ and $\eta$ are to be determined. From the integrability condition $d \omega^{c}+\omega_{a}^{c} \wedge \omega^{a}=0$, the following equations are obtained:

$$
\psi \xi=\chi_{x}, \quad \chi \eta=-\psi_{t} .
$$

Using (12), we obtain

$$
d(A \chi d t)+(\xi d t+\eta d x) \wedge C \psi d x=0
$$

which yields the equation

$$
A_{x} \chi+(A-C) \chi_{x}=0
$$

Similarly, from

$$
d(C \psi d x)+A \chi d t \wedge(\xi d t+\eta d x)=0
$$

it follows that

$$
C_{t} \psi+(C-A) \psi_{t}=0
$$


Since the surface is free of umbilics $A \neq \pm 1$, hence setting $A=\tanh (\alpha / 2)$ and $C=\operatorname{coth}(\alpha$ /2), these equations are transformed into

$$
(\ln \chi)_{x}=\left(\ln \cosh \frac{\alpha}{2}\right)_{x}, \quad(\ln \psi)_{t}=\left(\ln \sinh \frac{\alpha}{2}\right)_{t}
$$

hence $\chi(t)=f(t) \cosh (\alpha / 2)$ and $\psi(x)=g(x) \sinh (\alpha / 2)$. By means of a reparametrization of $x$ and $t$, we can write

$$
\chi=\cosh \frac{\alpha}{2}, \quad \psi=\sinh \frac{\alpha}{2} .
$$

Hence, from (19), it follows that

$$
\omega^{1}=\cosh \frac{\alpha}{2} d t, \quad \omega^{2}=\sinh \frac{\alpha}{2} d x
$$

This gives the required structure for the fundamental forms. The second part follows immediately from the Gauss equation, $d \omega_{2}^{1}=K \omega^{1} \wedge \omega^{2}$. Since

$$
\xi=\frac{\chi_{x}}{\psi}=\frac{1}{2} \alpha_{x}, \quad \eta=-\frac{\psi_{t}}{\chi}=-\frac{1}{2} \alpha_{t}
$$

The one-forms are given by

$$
\begin{gathered}
\omega^{1}=\cosh \frac{\alpha}{2} d t, \quad \omega^{2}=\sinh \frac{\alpha}{2} d x, \quad \omega_{2}^{1}=\frac{1}{2}\left(\alpha_{x} d t-\alpha_{t} d x\right), \\
\omega_{1}^{3}=\sinh \frac{\alpha}{2} d t, \quad \omega_{2}^{3}=\cosh \frac{\alpha}{2} d x .
\end{gathered}
$$

Differentiating $\omega_{2}^{1}$, it is found that

$$
d \omega_{2}^{1}=\xi_{x} d x \wedge d t+\eta_{t} d t \wedge d x=\left(\xi_{x}-\eta_{t}\right) d x \wedge d t=\frac{1}{2}\left(\alpha_{x x}+\alpha_{t t}\right) d x \wedge d t
$$

The right-hand side is $\omega^{1} \wedge \omega^{2}=-(1 / 2) \sinh \alpha d x \wedge d t$, thus when $K=-1$, we obtain the equation $\alpha_{x x}+\alpha_{t t}=\sinh \alpha$. The case $K=1$ follows similarly.

From these results, it follows that $A \chi=\sinh (\alpha / 2)$ and $C \psi=\cosh (\alpha / 2)$. Now, the equations for the surface provide a method for constructing a space-like surface of constant Gaussian curvature $K=-1$ in $\mathbb{R}^{2,1}$ which is based on a solution of the sinh-Laplace equation. This amounts to using these equations to write down a Lax pair for the system such that compatibility holds modulo the sinh-Laplace equation and which can be integrated given a particular solution of the equation.

Theorem 3. Let $\alpha(t, x) \neq 0$ be a solution of the sinh-Laplace equation (1) on a simply connected region $\Omega \subset \mathbb{R}^{2}$. Then there exists a space-like surface $S \subset \mathbb{R}^{2,1}$ of constant Gaussian curvature $K=-1$ such that $(t, x)$ are its Tschebyscheff coordinates.

Proof. If a solution for the set of basis vectors $\left\{\mathbf{e}_{1}, \mathbf{e}_{2}, \mathbf{e}_{3}\right\}$ can be obtained from the fundamental equations, then the equation for $d \mathbf{r}$ can be integrated to produce the coordinates 
of a surface. Therefore,

$$
d \mathbf{e}_{1}=\mathbf{e}_{1, x} d x+\mathbf{e}_{1, t} d t=\frac{1}{2} \alpha_{t} \mathbf{e}_{2} d x+\left(\sinh \frac{\alpha}{2} \mathbf{e}_{3}-\frac{1}{2} \alpha_{x} \mathbf{e}_{2}\right) d t
$$

Equating coefficients of $d x$ and $d t$ on both sides of (32), we obtain the pair

$$
\mathbf{e}_{1, x}=\frac{1}{2} \alpha_{t} \mathbf{e}_{2}, \quad \mathbf{e}_{1, t}=\sinh \frac{\alpha}{2} \mathbf{e}_{3}-\frac{1}{2} \alpha_{x} \mathbf{e}_{2}
$$

From the next equation, it follows that

$$
d \mathbf{e}_{2}=\mathbf{e}_{2, x} d x+\mathbf{e}_{2, t} d t=\frac{1}{2} \alpha_{x} \mathbf{e}_{1} d t+\left(-\frac{1}{2} \alpha_{t} \mathbf{e}_{1}+\cosh \frac{\alpha}{2} \mathbf{e}_{3}\right) d x
$$

Thus,

$$
\mathbf{e}_{2, x}=-\frac{1}{2} \alpha_{t} \mathbf{e}_{1}+\cosh \frac{\alpha}{2} \mathbf{e}_{3}, \quad \mathbf{e}_{2, t}=\frac{1}{2} \alpha_{x} \mathbf{e}_{1}
$$

Finally, the last equation gives the pair

$$
\mathbf{e}_{3, x}=\cosh \frac{\alpha}{2} \mathbf{e}_{2}, \quad \mathbf{e}_{3, t}=\sinh \frac{\alpha}{2} \mathbf{e}_{1} .
$$

To summarize these results, we introduce the more compact notation $\mathbf{e}_{1}=\mathbf{n}, \mathbf{e}_{2}=\mathbf{m}$, and $\mathbf{e}_{3}=\mathbf{p}$ such that $\mathbf{p}^{2}=-1, \mathbf{m}^{2}=\mathbf{n}^{2}=1, \mathbf{p} \cdot \mathbf{m}=\mathbf{p} \cdot \mathbf{n}=\mathbf{m} \cdot \mathbf{n}=0$, then this system takes the form

$$
\begin{gathered}
\mathbf{p}_{x}=\cosh \frac{\alpha}{2} \mathbf{m}, \quad \mathbf{p}_{t}=\sinh \frac{\alpha}{2} \mathbf{n}, \\
\mathbf{m}_{x}=-\frac{1}{2} \alpha_{t} \mathbf{n}+\cosh \frac{\alpha}{2} \mathbf{p}, \quad \mathbf{m}_{t}=\frac{1}{2} \alpha_{x} \mathbf{n}, \\
\mathbf{n}_{x}=\frac{1}{2} \alpha_{t} \mathbf{m}, \quad \mathbf{n}_{t}=-\frac{1}{2} \alpha_{x} \mathbf{m}+\sinh \frac{\alpha}{2} \mathbf{p} .
\end{gathered}
$$

Since system (2) is linear in $\left\{\mathbf{e}_{1}, \mathbf{e}_{2}, \mathbf{e}_{3}\right\}$, the orthonormal solution of (37) is defined on all of $\Omega$. To obtain the coordinates of the surface, it remains to integrate the expression for $d \mathbf{r}$, which is also integrable. For a solution of sinh-Laplace equation (1), system (37) is completely integrable, and the solution is defined on all of $\Omega$. Now an initial condition can be chosen $(t, x)=\left(t_{0}, x_{0}\right) \in \Omega$, and a surface is obtained in the coordinates of Definition 1 .

Lemma 4. The system (37) satisfies the necessary compatibility conditions provided that $\alpha(t, x)$ satisfies the sinh-Laplace equation $\alpha_{t t}+\alpha_{x x}=\sinh \alpha$.

Proof. The pair of equations $\left(\mathbf{p}_{x}, \mathbf{p}_{y}\right)$ is satisfied trivially with respect to (37). Differentiating $\mathbf{m}_{x}$ with respect to $t$, we obtain $\mathbf{m}_{x t}=-(1 / 2) \alpha_{t t} \mathbf{n}-(1 / 2) \alpha_{t} \mathbf{n}_{t}+\left(\alpha_{t} / 2\right) \sinh (\alpha / 2) \mathbf{p}+$ $\cosh (\alpha / 2) \mathbf{p}_{t}$, and $\mathbf{m}_{t}$ with respect to $x$ gives $\mathbf{m}_{t x}=(1 / 2) \alpha_{x x} \mathbf{n}+(1 / 2) \alpha_{x} \mathbf{n}_{x}$. Equating the 
derivatives $\mathbf{m}_{x t}=\mathbf{m}_{t x}$ modulo (37), we obtain

$$
-\frac{1}{2} \alpha_{t t} \mathbf{n}-\frac{1}{2} \alpha_{t}\left(-\frac{1}{2} \alpha_{x} \mathbf{m}+\sinh \frac{\alpha}{2} \mathbf{p}\right)+\frac{\alpha_{t}}{2} \sinh \frac{\alpha}{2} \mathbf{p}+\cosh \frac{\alpha}{2} \sinh \frac{\alpha}{2} \mathbf{n}=\frac{1}{2} \alpha_{x x} \mathbf{n}+\frac{1}{4} \alpha_{x} \alpha_{t} \mathbf{m} .
$$

Upon simplifying this expression, it is clear that it holds exactly when $\alpha$ satisfies the positive sinh-Laplace equation. A similar calculation gives the same result for the $\left(\mathbf{n}_{t}, \mathbf{n}_{x}\right)$ pair.

Theorem 5. Let $\alpha(t, x) \neq 0$ be a solution of the sinh-Laplace equation (1) on a simply connected region. Then there exists a space-like surface $S \subset \mathbb{R}^{3}$ of constant Gaussian curvature such that $(t, x)$ are the Tschebyscheff coordinates, and the associated Lax pair is given by

$$
\begin{gathered}
\mathbf{p}_{x}=\cosh \frac{\alpha}{2} \mathbf{m}, \quad \mathbf{p}_{t}=\sinh \frac{\alpha}{2} \mathbf{n}, \\
\mathbf{m}_{x}=-\frac{1}{2} \alpha_{t} \mathbf{n}-\cosh \frac{\alpha}{2} \mathbf{p}, \quad \mathbf{m}_{t}=\frac{1}{2} \alpha_{x} \mathbf{n}, \\
\mathbf{n}_{x}=\frac{1}{2} \alpha_{t} \mathbf{m}, \quad \mathbf{n}_{t}=-\frac{1}{2} \alpha_{x} \mathbf{m}-\sinh \frac{\alpha}{2} \mathbf{p} .
\end{gathered}
$$

Lemma 6. The system (39) satisfies the integrability or compatibility condition provided that $\alpha(t, x)$ satisfies the sinh-Laplace equation $\alpha_{t t}+\alpha_{x x}=-\sinh \alpha$.

It is worth explaining exactly how (1) is related to the Gaussian curvature $K$ of the actual surface. From (15), it can be seen that there is a very straightforward relationship between the Riemann tensor $R_{1212}$ and the Gaussian curvature of the corresponding surface. The metric for the cases of interest here is given for $\alpha \neq 0$ in Definition 1. Based on the metric, it is straightforward to calculate $R_{1212}$. In terms of $\alpha, R_{1212}$ is simply $(1 / 2)\left(\alpha_{t t}+\alpha_{x x}\right)$, hence (15) gives

$$
\frac{\left(\partial^{2} \alpha / \partial t^{2}\right)+\left(\partial^{2} \alpha / \partial x^{2}\right)}{-\sinh \alpha}=K
$$

For the case in which $K= \pm 1$, the sinh-Laplace equation (1) is obtained. This implies that if the metric of the unit sphere $S^{2}$ or $H^{2}$, for example, is written in the form (16), then $\alpha$ must be a solution of the sinh-Laplace system (1).

Consider the Lax pair given by (39). From the definition of the pair $\left(\mathbf{p}_{t}, \mathbf{p}_{x}\right)$, it is clear that

$$
\mathbf{p}_{x}^{2}-\mathbf{p}_{t}^{2}=1, \quad \mathbf{p}_{x} \cdot \mathbf{p}_{t}=0
$$

since $\mathbf{m}$ and $\mathbf{n}$ are orthonormal. Differentiating $\mathbf{p}_{t}$ with respect to $t$ and $\mathbf{p}_{x}$ with respect to $x$, we obtain

$$
\mathbf{p}_{t t}=\frac{\alpha_{t}}{2} \cosh \frac{\alpha}{2} \mathbf{n}+\sinh \frac{\alpha}{2} \mathbf{n}_{t}, \quad \mathbf{p}_{x x}=\frac{\alpha_{x}}{2} \sinh \frac{\alpha}{2} \mathbf{m}+\cosh \frac{\alpha}{2} \mathbf{m}_{x}
$$


Therefore, it follows by substituting the known derivatives from (39) that

$$
\begin{aligned}
\mathbf{p}_{t t}+\mathbf{p}_{x x}+\left(\mathbf{p}_{t}^{2}+\mathbf{p}_{x}^{2}\right) \mathbf{p}= & \frac{\alpha_{x}}{2} \sinh \frac{\alpha}{2} \mathbf{m}+\cosh \frac{\alpha}{2}\left(-\frac{1}{2} \alpha_{t} \mathbf{n}-\cosh \frac{\alpha}{2} \mathbf{p}\right)+\frac{\alpha_{t}}{2} \cosh \frac{\alpha}{2} \mathbf{n} \\
& +\sinh \frac{\alpha}{2}\left(-\frac{1}{2} \alpha_{x} \mathbf{m}-\sinh \frac{\alpha}{2} \mathbf{p}\right)+\left(\cosh ^{2} \frac{\alpha}{2}+\sinh ^{2} \frac{\alpha}{2}\right) \mathbf{p}=0 .
\end{aligned}
$$

To summarize this result, it has been shown that $\mathbf{p}(t, x)$ satisfies the equation

$$
\mathbf{p}_{t t}+\mathbf{p}_{x x}+\left(\mathbf{p}_{t}^{2}+\mathbf{p}_{x}^{2}\right) \mathbf{p}=0
$$

In fact, (44) is exactly the equation for harmonic maps from $\mathbb{R}^{2}$ to $S^{2}$. Consequently, $\mathbf{p}(t, x)$ is a harmonic map from $\mathbb{R}^{2}$ to $S^{2}$. A normalized harmonic map is defined as a harmonic map which satisfies (41) as well. Given a solution $\alpha(t, x)$ for the negative sinhLaplace equation (1) on a simply connected region $\Omega \subset \mathbb{R}^{2}$, by solving the Lax pair with some initial condition $\left(t_{0}, x_{0}\right) \in \Omega$, a normalized harmonic map is obtained. This implies that a normalized harmonic map can be constructed from a solution of the negative sinhLaplace equation (1). This establishes a connection between solutions of the negative sinh-Laplace equation and harmonic maps.

A surface of constant Gaussian curvature in $\mathbb{R}^{2,1}$ based on a particular solution of the sinh-Laplace equation corresponding to the harmonic maps from $\Omega \subset \mathbb{R}^{2}$ to $H^{2} \subset$ $\mathbb{R}^{2,1}$ will be obtained. Here $H^{2}$ is considered to be the upper component of $\mathbf{p}^{2}=-1$ in $\mathbb{R}^{2,1}$. The corresponding Lax pair that is integrated has been given in (37), and Lemma 4 clearly shows that integrability is satisfied provided that $\alpha$ is a solution of the positive sinh-Laplace equation. This is rigorously adhered to here. For purposes of presentation, the $t$ and $x$ labels will be interchanged in (37). A particularly nice form for the surface coordinates in which sinh $t$ and $\cosh t$ appear explicitly in terms of $t$ is obtained in this event, and the Lax takes the form

$$
\begin{gathered}
\mathbf{p}_{t}=\cosh \frac{\alpha}{2} \mathbf{m}, \quad \mathbf{p}_{x}=\sinh \frac{\alpha}{2} \mathbf{n}, \\
\mathbf{m}_{t}=-\frac{1}{2} \alpha_{x} \mathbf{n}+\cosh \frac{\alpha}{2} \mathbf{p}, \quad \mathbf{m}_{x}=\frac{1}{2} \alpha_{t} \mathbf{n}, \\
\mathbf{n}_{t}=\frac{1}{2} \alpha_{x} \mathbf{m}, \quad \mathbf{n}_{x}=-\frac{1}{2} \alpha_{t} \mathbf{m}+\sinh \frac{\alpha}{2} \mathbf{p} .
\end{gathered}
$$

The following $\alpha$ is a solution of the sinh-Laplace equation provided that $\mu^{2}+\lambda^{2}=1$,

$$
\alpha(t, x)=2 \sinh ^{-1}\left(\frac{1}{\sinh (\lambda t+\mu x)}\right), \quad \lambda t+\mu x>0 .
$$

This implies that

$$
\sinh \frac{\alpha}{2}=\frac{1}{\sinh (\lambda t+\mu x)}, \quad \cosh \frac{\alpha}{2}=\frac{\cosh (\lambda t+\mu x)}{\sinh (\lambda t+\mu x)}
$$


This solution will be substituted into the Lax pair given by (37) and integrated. To this end, differentiating (47), it follows that the derivatives of $\alpha$ are

$$
\alpha_{x}=-2 \mu \sinh \frac{\alpha}{2}, \quad \alpha_{t}=-2 \lambda \sinh \frac{\alpha}{2} .
$$

We introduce three new variables $\mathbf{a}, \mathbf{b}$, and $\mathbf{c}$ which are defined by

$$
\mathbf{a}=\lambda \mathbf{p}+\mathbf{m}, \quad \mathbf{b}=\mathbf{p}+\lambda \mathbf{m}+\mu \mathbf{n}, \quad \mathbf{c}=-\mathbf{p}-\lambda \mathbf{m}+\mu \mathbf{n} .
$$

Differentiating a with respect to $x$, it follows with respect to the Lax pair that

$$
\mathbf{a}_{x}=\lambda \mathbf{p}_{x}+\mathbf{m}_{x}=\lambda \sinh \frac{\alpha}{2} \mathbf{n}-\lambda \sinh \frac{\alpha}{2} \mathbf{n}=0 .
$$

Similarly, differentiating $\mathbf{b}$ and $\mathbf{c}$ with respect to $x$, then modulo the Lax, we obtain that $\mathbf{a}, \mathbf{b}$, and $\mathbf{c}$ satisfy the following system:

$$
\mathbf{a}_{x}=0, \quad \mathbf{b}_{x}=\mu \sinh \frac{\alpha}{2} \mathbf{b}, \quad \mathbf{c}_{x}=-\mu \sinh \frac{\alpha}{2} \mathbf{c} .
$$

This system of differential equations may be integrated to yield

$$
\mathbf{a}(x, t)=\mathbf{a}_{0}(t), \quad \mathbf{b}(x, t)=-\mathbf{b}_{0}(t) \tanh \left(\frac{\lambda t+\mu x}{2}\right), \quad \mathbf{c}(x, t)=-\mathbf{c}_{0}(t) \operatorname{coth}\left(\frac{\lambda t+\mu x}{2}\right),
$$

where $\mathbf{b}$ and $\mathbf{c}$ can be expressed in terms of $\alpha$ as follows:

$$
\mathbf{b}(x, t)=-\mathbf{b}_{0}(t)\left(\cosh \frac{\alpha}{2}-\sinh \frac{\alpha}{2}\right), \quad \mathbf{c}(x, t)=-\mathbf{c}_{0}(t)\left(\cosh \frac{\alpha}{2}+\sinh \frac{\alpha}{2}\right) .
$$

Here $\mathbf{a}_{0}(t), \mathbf{b}_{0}(t)$, and $\mathbf{c}_{0}(t)$ are vector functions which are independent of $x$. Moreover, $\mathbf{p}$, $\mathbf{m}$, and $\mathbf{n}$ can be determined in terms of $\mathbf{a}, \mathbf{b}$, and $\mathbf{c}$ as follows:

$$
\mathbf{p}=\frac{1}{2 \mu^{2}}(\mathbf{b}-2 \lambda \mathbf{a}-\mathbf{c}), \quad \mathbf{m}=\frac{1}{2 \mu^{2}}(2 \mathbf{a}+\lambda \mathbf{c}-\lambda \mathbf{b}), \quad \mathbf{n}=\frac{1}{2 \mu}(\mathbf{b}+\mathbf{c}) .
$$

Next, a system of equations which are satisfied by $\mathbf{a}_{0}, \mathbf{b}_{0}$, and $\mathbf{c}_{0}$ will be obtained and which can be solved. Differentiating a with respect to $t$ and using the Lax as well as (53), we obtain

$$
\begin{aligned}
\mathbf{a}_{t} & =\lambda \mathbf{p}_{t}+\mathbf{m}_{t}=\cosh \frac{\alpha}{2}(\lambda \mathbf{m}+\mathbf{p})+\mu \sinh \frac{\alpha}{2} \mathbf{n}=\frac{1}{2} \cosh \frac{\alpha}{2}(\mathbf{b}-\mathbf{c})+\frac{1}{2} \sinh \frac{\alpha}{2}(\mathbf{b}+\mathbf{c}) \\
& =-\frac{1}{2} \mathbf{b}_{0}\left(\cosh ^{2} \frac{\alpha}{2}-\sinh ^{2} \frac{\alpha}{2}\right)-\frac{1}{2} \mathbf{c}_{0}\left(\sinh ^{2} \frac{\alpha}{2}-\cosh ^{2} \frac{\alpha}{2}\right)
\end{aligned}
$$

which simplifies to

$$
\mathbf{a}_{0 t}=-\frac{1}{2} \mathbf{b}_{0}+\frac{1}{2} \mathbf{c}_{0}
$$


Similar operations can be performed on $\mathbf{b}$ and $\mathbf{c}$, and the system which is satisfied by $\mathbf{a}_{0}$, $\mathbf{b}_{0}$, and $\mathbf{c}_{0}$ is given by

$$
\mathbf{a}_{0 t}=-\frac{1}{2} \mathbf{b}_{0}+\frac{1}{2} \mathbf{c}, \quad \mathbf{b}_{0 t}=-\mathbf{a}_{0}, \quad \mathbf{c}_{0 t}=\mathbf{a}_{0} .
$$

Differentiating the first equation with respect to $t$, then replacing the last two, we find that a satisfies $\mathbf{a}_{0 t t}=\mathbf{a}_{0}$, so the general solution of this system is given by

$$
\begin{aligned}
& \mathbf{a}_{0}(t)=\boldsymbol{\sigma} \cosh t+\boldsymbol{\nu} \sinh t, \\
& \mathbf{b}_{0}(t)=\boldsymbol{\tau}-\boldsymbol{\sigma} \sinh t-\boldsymbol{\nu} \cosh t, \\
& \mathbf{c}_{0}(t)=\boldsymbol{\tau}+\boldsymbol{\sigma} \sinh t+\boldsymbol{\nu} \cosh t
\end{aligned}
$$

in terms of constant vectors $\tau, \sigma$, and $\boldsymbol{\nu}$. Let $t=0$ and $x \rightarrow-\infty$, then from (58), we obtain

$$
\begin{gathered}
\mathbf{a}_{0}(0)=\boldsymbol{\sigma}, \quad \mathbf{b}_{0}(0)=\boldsymbol{\tau}-\boldsymbol{\nu}, \quad \mathbf{c}_{0}(0)=\boldsymbol{\tau}+\boldsymbol{\nu}, \\
\mathbf{a}_{0}(0)=\lambda \mathbf{p}_{0}-\mathbf{m}_{0}, \quad \mathbf{b}_{0}(0)=\mathbf{p}_{0}+\lambda \mathbf{m}_{0}+\mu \mathbf{n}_{0}, \quad \mathbf{c}_{0}(0)=-\mathbf{p}_{0}-\lambda \mathbf{m}_{0}+\mu \mathbf{n}_{0} .
\end{gathered}
$$

Eliminating $\mathbf{a}_{0}(0), \mathbf{b}_{0}(0)$, and $\mathbf{c}_{0}(0)$, we obtain

$$
\boldsymbol{\sigma}=\lambda \mathbf{p}_{0}-\mathbf{m}_{0}, \quad \boldsymbol{\tau}-\boldsymbol{v}=\mathbf{p}_{0}+\lambda \mathbf{m}_{0}+\mu \mathbf{n}_{0}, \quad \boldsymbol{\tau}+\boldsymbol{\nu}=-\mathbf{p}_{0}-\lambda \mathbf{m}_{0}+\mu \mathbf{n}_{0} .
$$

Adding the second and third equations, we find that $\boldsymbol{\tau}=\mu \mathbf{n}_{0}$, and then subtracting, we have

$$
\boldsymbol{\nu}=-\mathbf{p}_{0}-\lambda \mathbf{m}_{0}
$$

Here, $\mathbf{p}_{0}, \mathbf{m}_{0}$ and $\mathbf{n}_{0}$ form an orthonormal set of vectors in $H^{2} \subset \mathbb{R}^{2,1}$. Substituting $\boldsymbol{\tau}, \boldsymbol{\sigma}$ and $\boldsymbol{v}$ into the expression for $\mathbf{a}_{0}(t), \mathbf{b}_{0}(t)$, and $\mathbf{c}_{0}(t)$, we obtain

$$
\begin{aligned}
& \mathbf{a}_{0}(t)=\left(\lambda \mathbf{p}_{0}-\mathbf{m}_{0}\right) \cosh t-\left(\mathbf{p}_{0}+\lambda \mathbf{m}_{0}\right) \sinh t \\
& \mathbf{b}_{0}(t)=\mu \mathbf{n}_{0}-\left(\lambda \mathbf{p}_{0}-\mathbf{m}_{0}\right) \sinh t+\left(\mathbf{p}_{0}+\lambda \mathbf{m}_{0}\right) \cosh t \\
& \mathbf{c}_{0}(t)=\mu \mathbf{n}_{0}+\left(\lambda \mathbf{p}_{0}-\mathbf{m}_{0}\right) \sinh t-\left(\mathbf{p}_{0}+\lambda \mathbf{m}_{0}\right) \cosh t .
\end{aligned}
$$

Substituting these results into (54), we obtain that with $\zeta=\mu x+\lambda t$, and collecting all terms which multiply $\mathbf{p}_{0}, \mathbf{m}_{0}$, and $\mathbf{n}_{0}$, respectively, we can write $\mathbf{p}, \mathbf{m}$, and $\mathbf{n}$ explicitly as follows:

$$
\begin{aligned}
\mathbf{p}= & \left(\frac{\lambda}{\mu^{2}}(\sinh t-\lambda \cosh t)+(\lambda \sinh t-\cosh t) \operatorname{coth} \zeta\right) \mathbf{p}_{0}+\frac{\mathbf{n}_{0}}{\mu \sinh \zeta} \\
& +\left(-\frac{1}{\mu^{2}}(\lambda \cosh t+\sinh t) \operatorname{coth} \zeta+\frac{\lambda}{\mu^{2}}(\cosh t+\lambda \sinh t)\right) \mathbf{m}_{0}, \\
\mathbf{m}= & \left(\frac{1}{\mu^{2}}(\lambda \cosh t-\sinh t) \sinh \zeta+\frac{\lambda}{\mu^{2}} \operatorname{coth} \zeta(\lambda \sinh t-\cosh t)\right) \mathbf{p}_{0}-\frac{\lambda \mathbf{n}_{0}}{\mu \sinh \zeta} \\
& -\left(\frac{1}{\mu^{2}}(\cosh t+\lambda \sinh t)-\frac{\lambda}{\mu^{2}} \operatorname{coth} \zeta(\lambda \cosh t+\sinh t)\right) \mathbf{m}_{0}, \\
\mathbf{n}= & \frac{(\cosh t-\lambda \sinh t)}{\mu^{2} \sinh \zeta} \mathbf{p}_{0}+\frac{(\sinh t+\lambda \cosh t)}{\mu^{2} \sinh \zeta} \mathbf{m}_{0}-\frac{1}{\mu} \operatorname{coth} \zeta \mathbf{n}_{0} .
\end{aligned}
$$


To obtain one variety of surface, it suffices to take $d \mathbf{r}$ in the form

$$
d \mathbf{r}=\cosh \frac{\alpha}{2} d t \mathbf{m}+\sinh \frac{\alpha}{2} d x \mathbf{n}
$$

This clearly generates the correct form $I=d \mathbf{r} \cdot d \mathbf{r}$. In this form, it is possible to do all the integration. Integrating $\mathbf{r}_{x}=\sinh (\alpha / 2) \mathbf{n}$ with respect to $x$ by taking $\mathbf{n}$ from (63), we obtain

$$
\mathbf{r}=\mathbf{r}_{0}(t)-\frac{1}{\mu^{2}}(\sinh t-\lambda \cosh t) \operatorname{coth} \zeta \mathbf{m}_{0}+\frac{1}{\mu \sinh \zeta} \mathbf{n}_{0}+\frac{1}{\mu^{2}}(\lambda \sinh t-\cosh t) \operatorname{coth} \zeta \mathbf{p}_{0}
$$

To determine the remaining function $\mathbf{r}_{0}(t)$, differentiate (65) with respect to $t$ and substitute $\mathbf{r}_{t}$ and $\mathbf{m}$ into the remaining equation $\mathbf{r}_{t}=\cosh (\alpha / 2) \mathbf{m}$. This function satisfies the first-order equation

$$
\mathbf{r}_{0}^{\prime}(t)=\frac{\lambda}{\mu^{2}}(\cosh t-\lambda \sinh t) \mathbf{p}_{0}+\frac{\lambda}{\mu^{2}}(\sinh t+\lambda \cosh t) \mathbf{m}_{0}
$$

Integrating both sides of this, we finally obtain that

$$
\mathbf{r}_{0}(t)=\frac{\lambda}{\mu^{2}}(\sinh t-\lambda \cosh t) \mathbf{p}_{0}+\frac{\lambda}{\mu^{2}}(\cosh t+\lambda \sinh t) \mathbf{m}_{0}
$$

Substituting (67) into (65), we obtain the final equation for $\mathbf{r}$,

$$
\begin{aligned}
\mathbf{r}= & \frac{1}{\mu^{2}}(\lambda(\sinh t-\lambda \cosh t)+(\lambda \sinh t-\cosh t) \operatorname{coth} \zeta) \mathbf{p}_{0}+\frac{\mathbf{n}_{0}}{\mu \sinh \zeta} \\
& +\frac{1}{\mu^{2}}(\lambda(\cosh t+\lambda \sinh t)-(\sinh t+\lambda \cosh t) \operatorname{coth} \zeta) \mathbf{m}_{0} .
\end{aligned}
$$

This result gives an expression for the coordinates of the surface in this space.

\section{References}

[1] P. Bracken and A. M. Grundland, Symmetry properties and explicit solutions of the generalized Weierstrass system, J. Math. Phys. 42 (2001), no. 3, 1250-1282.

[2] - On complete integrability of the generalized Weierstrass system, J. Nonlinear Math. Phys. 9 (2002), no. 2, 229-247.

[3] P. Bracken, A. M. Grundland, and L. Martina, The Weierstrass-Enneper system for constant mean curvature surfaces and the completely integrable sigma model, J. Math. Phys. 40 (1999), no. 7, 3379-3403.

[4] A. Das, Integrable Models, World Scientific Lecture Notes in Physics, vol. 30, World Scientific, New Jersy, 1989. 


\section{Determination of surfaces}

[5] H. S. Hu, Sine-Laplace equation, sinh-Laplace equation and harmonic maps, Manuscripta Math. 40 (1982), no. 2-3, 205-216.

[6] The construction of hyperbolic surfaces in 3-dimensional Minkowski space and sinhLaplace equation, Acta Math. Sinica (N.S.) 1 (1985), no. 1, 79-86.

Paul Bracken: Department of Mathematics, University of Texas - Pan American, Edinburg, TX 78541-2999, USA

E-mail address: bracken@panam.edu 


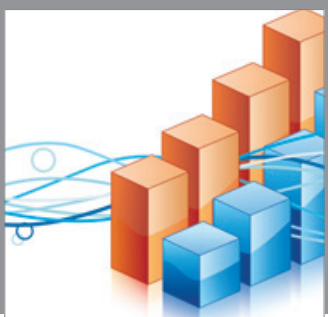

Advances in

Operations Research

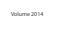

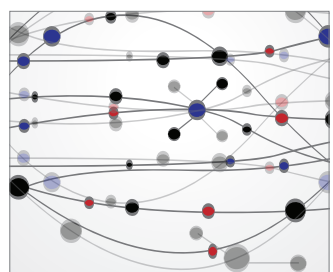

\section{The Scientific} World Journal
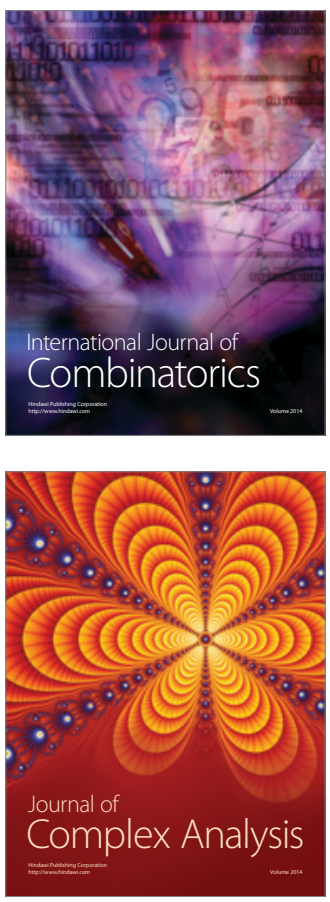

International Journal of

Mathematics and

Mathematical

Sciences
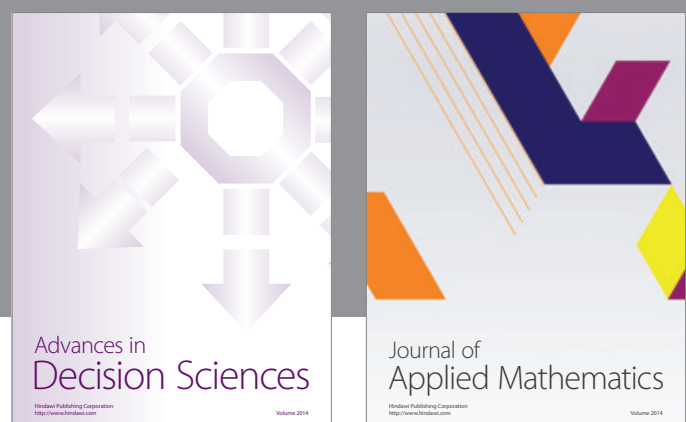

Journal of

Applied Mathematics
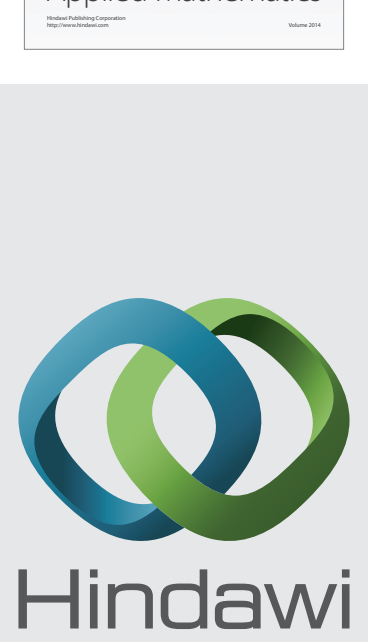

Submit your manuscripts at http://www.hindawi.com
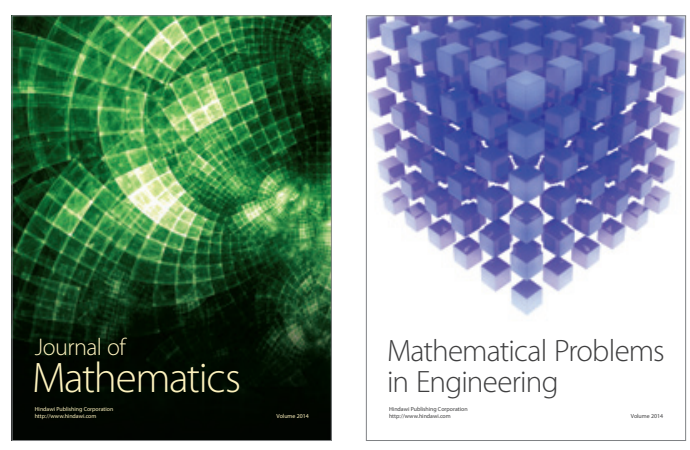

Mathematical Problems in Engineering
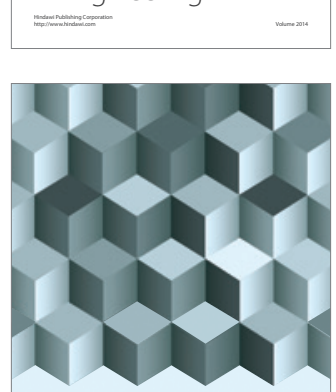

Journal of

Function Spaces
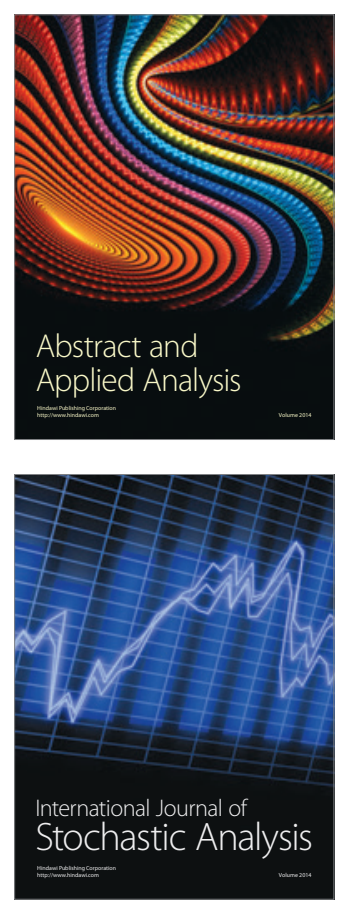

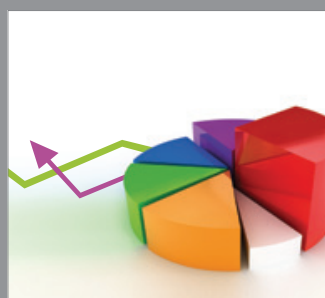

ournal of

Probability and Statistics

Promensencen
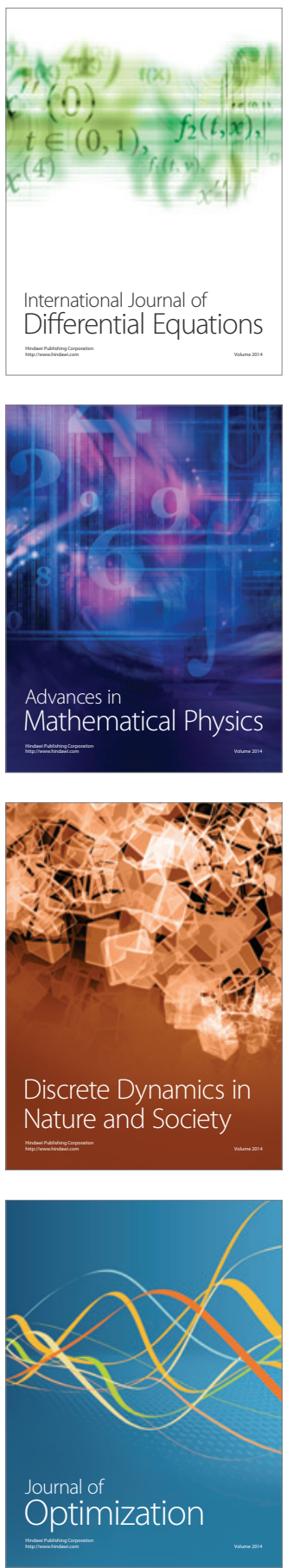